\title{
Teaching NeuroImages: An aTIPICal cause of acute neck pain
}

Stephanie Gosselin-Lefebvre, MD, FRCPC, Athari K. Salmeen, MD, FRCPC, Danielle Byrne, MD, RCSI, Jason R. Shewchuk, MD, FRCPC, and Thalia S. Field, MD, FRCPC, MHSC

Neurology ${ }^{\circledR}$ 2020;95:e1112-e1113. doi:10.1212/WNL.0000000000010094

Figure 1 Initial imaging

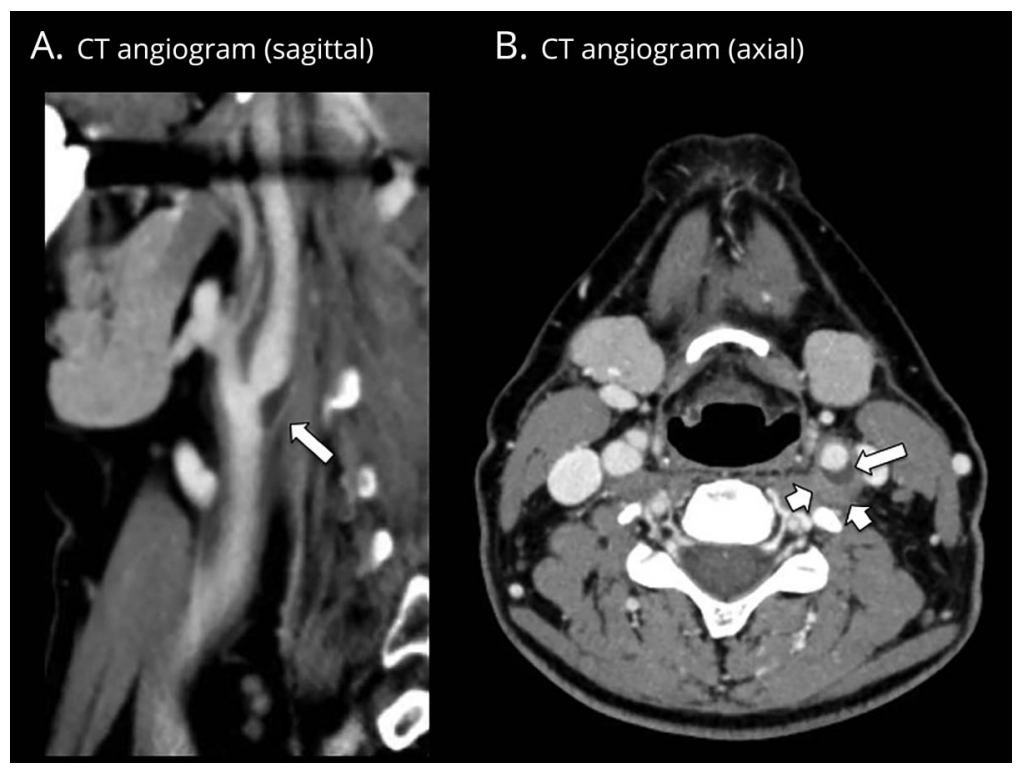

(A) Sagittal and (B) axial CT angiography images demonstrate eccentric low-attenuation plaque along the posterior wall of the distal left common carotid artery (long arrow) without significant luminal narrowing. Surrounding soft tissue and fat stranding (short arrows) are consistent with perivascular inflammation.

A healthy 54-year-old woman developed spontaneous acute onset of sharp left-sided neck and facial pain and odynophagia following a brief flu-like illness. CT angiography revealed eccentric perivascular infiltration at the left carotid bifurcation (figure 1) with corresponding enhancement on MRI (figure 2). She was empirically treated with prednisone and had clinical and imaging improvement at follow-up.

Transient perivascular inflammation of the carotid artery (TIPIC) is a rare, self-limited entity distinguishable on vascular imaging from extracranial dissection by lack of luminal narrowing and marked perivascular inflammation. ${ }^{1}$ Reported treatments include nonsteroidal antiinflammatory drugs, antiplatelet agents, and steroids. There is no known associated risk of stroke. Pain typically resolves within 2 weeks, with radiologic improvement by 3 months. ${ }^{2}$

\author{
Correspondence \\ Dr. Gosselin-Lefebvre \\ stephanie.gosselin- \\ lefebvre.1@ulaval.ca
}

\section{Study funding}

No targeted funding reported.

\section{MORE ONLINE}

$\rightarrow$ Teaching slides

links.lww.com/WNL/ B145

From Neurology (S.G.-L.), Université Laval, Québec, Canada; Jaber Al-Ahmad Al Sabah Hospital (A.K.S.), Kuwait City, Kuwait; and Radiology (D.B., J.R.S.) and Neurology (T.S.), University of British Columbia, Vancouver, Canada.

Go to Neurology.org/N for full disclosures. Funding information and disclosures deemed relevant by the authors, if any, are provided at the end of the article. 


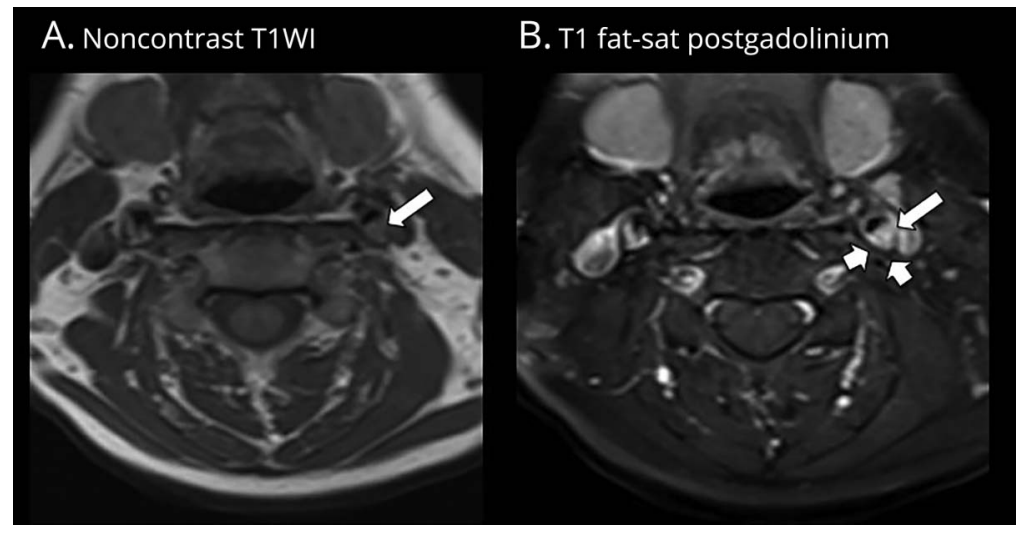

(A) Noncontrast T1-weighted axial MRI at 3 weeks post-CT showing minimally hyperintense plaque at the posterior aspect of the distal left common carotid artery (CCA), which enhances post IV contrast on (B) postgadolinium T1 fat-saturation imaging axial MRI (long arrows). There is a clear fat plane at the posterior margin of the left CCA (short arrows) consistent with resolution of perivascular inflammation, which was identified on CT at the time of initial presentation.

\section{Disclosure}

The authors report no disclosures relevant to the manuscript. Go to Neurology.org/N for full disclosures.

\section{Appendix Authors}

\begin{tabular}{|c|c|c|}
\hline Name & Location & Contribution \\
\hline $\begin{array}{l}\text { Stephanie } \\
\text { Gosselin- } \\
\text { Lefebvre, MD, } \\
\text { FRCPC }\end{array}$ & $\begin{array}{l}\text { Université Laval, } \\
\text { Québec, Canada }\end{array}$ & $\begin{array}{l}\text { Gathered patient data, } \\
\text { drafted manuscript, } \\
\text { revised figure captions }\end{array}$ \\
\hline $\begin{array}{l}\text { Athari K. } \\
\text { Salmeen, MD, } \\
\text { FRCPC }\end{array}$ & $\begin{array}{l}\text { Jaber Al-Ahmad Al } \\
\text { Sabah Hospital, } \\
\text { Kuwait City, Kuwait }\end{array}$ & $\begin{array}{l}\text { Gathered patient data, } \\
\text { revised manuscript }\end{array}$ \\
\hline $\begin{array}{l}\text { Danielle Byrne, } \\
\text { MD, RCSI }\end{array}$ & $\begin{array}{l}\text { Vancouver General } \\
\text { Hospital, Canada }\end{array}$ & $\begin{array}{l}\text { Prepared figures, drafted } \\
\text { figure captions, revised } \\
\text { manuscript }\end{array}$ \\
\hline
\end{tabular}

Appendix (continued)

\begin{tabular}{lll}
\hline Name & Location & Contribution \\
\hline $\begin{array}{l}\text { Jason } \\
\text { Shewchuk, MD, }\end{array}$ & $\begin{array}{l}\text { Vancouver General } \\
\text { Hospital, Canada }\end{array}$ & $\begin{array}{l}\text { Prepared figures, revised } \\
\text { figure captions and } \\
\text { manuscript }\end{array}$ \\
\hline $\begin{array}{l}\text { Thalia S. Field, } \\
\text { MD, FRCPC, }\end{array}$ & $\begin{array}{l}\text { Vancouver General } \\
\text { MHSc }\end{array}$ & $\begin{array}{l}\text { Conception, revised } \\
\text { manuscript and figure } \\
\text { captions }\end{array}$ \\
\hline
\end{tabular}

\section{References}

1. Lecler A, Obadia M, Savatovsky J, et al. TIPIC syndrome: beyond the myth of Carotidynia, a new distinct unclassified entity. Am J Neuroradiology 2017;38: 1391-1398.

2. Ulus S, Aksoy Ozcan U, Arsian A, et al. Imaging spectrum of TIPIC syndrome. Clin Neuroradiol Epub 2018 Nov 23. 


\title{
Neurology
}

\author{
Teaching NeuroImages: An aTIPICal cause of acute neck pain \\ Stephanie Gosselin-Lefebvre, Athari K. Salmeen, Danielle Byrne, et al. \\ Neurology 2020;95; e1112-e1113 Published Online before print June 30, 2020 \\ DOI 10.1212/WNL.0000000000010094
}

This information is current as of June 30, 2020

Updated Information \&
Services

References

Subspecialty Collections

Permissions \& Licensing

Reprints including high resolution figures, can be found at: http://n.neurology.org/content/95/8/e1112.full

This article cites 1 articles, 1 of which you can access for free at: http://n.neurology.org/content/95/8/e1112.full\#ref-list-1

This article, along with others on similar topics, appears in the following collection(s):

Clinical neurology history

http://n.neurology.org/cgi/collection/clinical_neurology_history MRI

http://n.neurology.org/cgi/collection/mri

Other cerebrovascular disease/ Stroke

http://n.neurology.org/cgi/collection/other_cerebrovascular_disease_s troke

Secondary headache disorders

http://n.neurology.org/cgi/collection/secondary_headache_disorders

Information about reproducing this article in parts (figures,tables) or in its entirety can be found online at:

http://www.neurology.org/about/about_the_journal\#permissions

Information about ordering reprints can be found online:

http://n.neurology.org/subscribers/advertise

Neurology ${ }^{\circledR}$ is the official journal of the American Academy of Neurology. Published continuously since 1951, it is now a weekly with 48 issues per year. Copyright () 2020 American Academy of Neurology. All rights reserved. Print ISSN: 0028-3878. Online ISSN: 1526-632X.

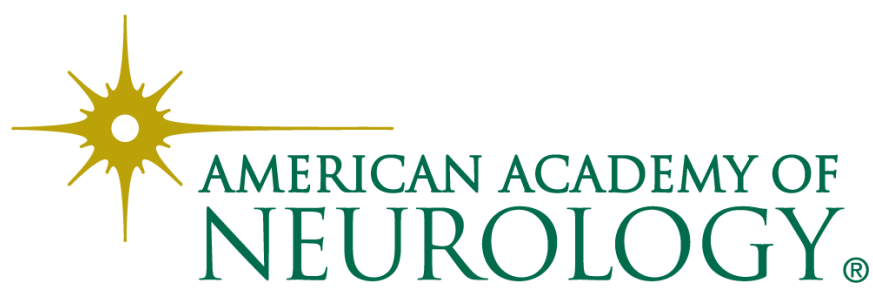

